\title{
A Quantitative Measure, Mechanism and Attractor for Self-Organization in Networked Complex Systems
}

\author{
Georgi Yordanov Georgiev \\ Department of Natural Sciences - Physics and Astronomy, Assumption College, \\ 500 Salisbury St, Worcester MA, 01609, United States of America \\ ggeorgie@assumption.edu, georgi@alumni.tufts.edu
}

\begin{abstract}
Quantity of organization in complex networks here is measured as the inverse of the average sum of physical actions of all elements per unit motion multiplied by the Planck's constant. The meaning of quantity of organization is the number of quanta of action per one unit motion of an element. This definition can be applied to the organization of any complex system. Systems self-organize to decrease the average action per element per unit motion. This lowest action state is the attractor for the continuous self-organization and evolution of a dynamical complex system. Constraints increase this average action and constraint minimization by the elements is a basic mechanism for action minimization. Increase of quantity of elements in a network, leads to faster constraint minimization through grouping, decrease of average action per element and motion and therefore accelerated rate of self-organization. Progressive development, as self-organization, is a process of minimization of action.
\end{abstract}

Keywords: network, self-organization, complex system, organization, quantitative measure, principle of least action, principle of stationary action, attractor, progressive development, acceleration.

\section{$1 \quad$ Introduction}

\subsection{Motivation}

To define quantitatively self-organization in complex networked systems a measure for organization is necessary [1]. Two systems should be numerically distinguishable by their degree of organization and their rate of self-organization. What one quantity can measure the degree of self-organization in all complex systems? To answer this question we turn to established science principles and ask: What single principle can explain the largest number of science phenomena? It turns out that this is the principle of least (stationary) action. There is no more broad and fundamental principle in science than this, as it can be seen in the next section.

\subsection{The Principle of Least Action and Its Variations}

Pierre de Maupertuis stated Law of the Least Action as a "universal principle from which all other principles naturally flow" [2]. Later Euler, Lagrange, Hamilton, 
Fermat, Einstein, and many others refined it and applied it to develop all areas of physics [3]. It was later generalized as a path integral formalism for quantum mechanics by Feynman [4]. Jacobi's form of the principle refers to the path of the system point in a curvilinear space characterized by the metric tensor [3]. The Hertz's principle of least curvature says that a particle tends to travel along the path with least curvature, if there are not external forces acting on it [3]. The Gauss Principle of least constraint where the motion of a system of interconnected material points is such as to minimize the constraint on the system is an alternative formulation of classical mechanics, using a differential variational principle [5]. Action is more general than energy and any law derived from the principle of least action is guaranteed to be self consistent [6]. All of the laws of motion and conservation in all branches of physics are derived from the principle of least action $[6,7]$.

\subsection{Applications to Networks and Complex Systems}

Scientists have applied the principle of least action to networks and complex systems. For example, it has been applied to network theory $[8,9,10]$ and path integral approaches to stochastic processes and networks [11]. Samples of some other applications are by Annila and Salthe for natural selection [12] and Devezas for technological change [13]. Some of the other important measures and methods used in complex systems research are presented by Chaisson [14], Bar-Yam [15], Smart [16], Vidal [17] and Gershenson and Heylighen [18]. This list is not exhaustive. Some of these established measures use information, entropy or energy to describe complexity, while a fundamental quantity of physical action is used in this work to describe degree of organization through efficiency.

\section{Principle of Least Action for a System of Elements - An Attractor}

In a previous paper [1] we defined the natural state of an organized system as the one in which the variation of the sum of actions of all of the elements is zero. Here we define the principle of least action for $\mathrm{n}$ elements crossing m nodes as:

$$
\delta \sum_{i=0}^{n} \sum_{j=0}^{m} I_{i j}=\delta \sum_{i=1}^{n} \sum_{j=1}^{m} \int_{t_{1}}^{t_{2}} L_{i j} d t=0
$$

Where $\delta$ is infinitesimally small variation in the action integral $I_{i j}$ of the $\mathrm{j}^{\text {th }}$ crossings between the nodes (unit motion) of the $\mathrm{i}^{\text {th }}$ element and $L_{i j}$ is the Lagrangian for that motion. $n$ represents the number of elements in a system, $\mathrm{m}$ the number of motions and $t_{l}$ and $t_{2}$ are the initial and final times of each motion. $\sum_{i=0}^{n} \sum_{j=0}^{m} I_{i j}$ is the sum of all actions of all elements $\mathrm{n}$ for their motions $\mathrm{m}$ between nodes of a complex network. For example, a unit motion for electrons on a computer chip is the one necessary for one computation. For a computer network, such as internet, it is the transmission of one bit of information. In a chemical system it is the one for one chemical reaction. The state of zero variation of the total action for all motions is the one to which any system is naturally driven. Open systems never achieve this least action state because 
of the constant changes that occur in them, but are always tending toward it. In some respect one can consider this attractor state to be one of dynamical action equilibrium. Using the quantity of action one can measure how far the system is from this equilibrium and can distinguish between the organizations of two systems, both of which are equally close to equilibrium.

\section{Physical Action as a Quantitative Measure for Organization}

In [1] we defined organization of a system as inversely proportional to the average sum of all actions. Here we expand this notion by defining organization, $\alpha$, as inversely proportional to the average action per one element and one motion.

$$
\alpha=\frac{h n m}{\sum_{i=0}^{n} \sum_{j=0}^{m} I_{i j}}
$$

$\mathrm{h}$ is the Planck's constant. The meaning of organization is that it is inversely proportional to the number of quanta of action per one motion of one element in a system. This definition is for a system of identical elements or where elements can be approximated as identical. It is the efficiency of physical action. The time derivative of $\alpha$ is the rate of progressive development of a complex system.

\section{$4 \quad$ Applications}

\subsection{One Element and One Constraint}

Consider the simplest possible part of a network: one edge, two nodes and one element moving from node 1 to node 2 . Let's consider case (I) when there is no constraint for the motion of the element. It crosses the path between nodes 1 and 2 along the shortest line - a geodesic. Now consider case (II) when there is one constraint placed between nodes 1 and 2 and the shortest path of the element in this case is not a geodesic. If the path is twice as long in the second case, if the kinetic energy of the element is the same as in case (I) and no potentials are present, then the time taken to cross between nodes 1 and 2 is twice as long. Therefore the action in case (II) is twice than the action in case (I). When we substitute these numbers in the expression for organization $\alpha$ (eq. 2), where $n=1$, one element, and $m=1$, one crossing between two nodes, then the denominator which is just the action of the element for that motion will be twice as large in the second case and therefore the result for the amount of organization is a half as compared to the first case.

\subsection{Many Elements and Constraints}

Now consider an arbitrary networks consisting of three, ten, thousands, millions and billions of nodes and edges, populated by as many elements and constraints, where the paths of the elements cross each other. The optimum of all of the constraints', nodes', edges' and elements' positions and the motions of the elements is the 
minimum possible action state of the entire system, providing a numerical measure for its organization. Notice that action is not at an absolute possible minimum in this case, but at a higher, optimal value. Action would be at its absolute minimum only in a system without any constraints on the motion of its elements, which is not the case in complex systems and networks. Nevertheless, action is at a minimum compared to what it will be for all other arrangements of nodes, elements and constraints in the system that are less organized. When we consider an open dynamical system, where the number and positions of nodes, edges, elements and constraints constantly changes, then this minimum action state is constantly recalculated by the system. It is an attractor state which drives the system to higher level of organization and this process can continue indefinitely, as long as the system exists. Achieving maximum organization is a dynamical process in open complex systems of constantly recalculating positions of nodes, edges, elements and constraints for a least action state and preserving those positions in a physical memory of the organization of the system.

\section{Exploring the Limits for Organization}

\subsection{An Upper Limit}

The smallest possible discrete amount of action is one quantum of it, equal to the value of the Planck's constant. With self-organization the distances between the nodes shrink, the elements become smaller and the constraints for their motion decrease, for the purpose of decreasing of action (as in computer chips). The limit for this process of decrease of action is one quantum of it. If each motion uses the minimum of one quantum of action, then the value of the organization, $\alpha$, is exactly one.

Can this value for organization be exceeded by a parallel processes, like quantum computing, where possibly with one, or a few quanta of action a vast number of computations can occur? Technically the crossing is still between two nodes, but it happens simultaneously along infinite number of different paths. It is like an infinite number of elements crossing between two nodes, each performing different computations. Alternatively, with decrease of the amount of action per crossing, it might be possible for the elements to cross several nodes (do several motions) with one quantum of action. In both of these cases the upper limit for organization, $\alpha$, becomes very large and possibly infinity.

\subsection{A Lower Limit}

For a completely disorganized system, where the entropy is at a maximum, all points in the system are equally probable for an element to visit. In order to reach its final destination, an element of the system must visit all points in it (by definition for maximum entropy), thus maximizing its action for one crossing from any node 1 to any node 2 . In this case, the action is extremely large and the organization, $\alpha$, of this system is very close to zero.

Another way to describe the lower limit for organization of a system is when the constraint for the motion between nodes 1 and 2 is infinitely large and the path taken 
by the element to cross between the nodes is infinitely long. This also maximizes action and describes a completely disorganized system. The value for organization, $\alpha$, in this case again approaches a limit of zero.

\section{Mechanism of Self-Organization}

When elements interact with constraints they apply force to minimize them, lowering their action for the next cycle. With the increase of quantity in a system, several elements can group on the same constraint to minimize it for less time. Decreased average action makes a system more stable, by lowering the energy needed for each motion. High average action, in disorganized system destabilizes it and above some limit it falls apart. Therefore a system with low enough average action can increase its quantity within limits of stability. Quantity and level of organization are proportional. If the quantity becomes constant, then the organization will reach a least action state and stop increasing. For continued self-organization an increase of the quantity is necessary. Quantity and level of organization of a system are in an accelerating positive feedback loop, ensuring unlimited increase of the level of organization in a system, unless it is destroyed by external influence, like limited resources, huge influx of energy, force impact, change in the conditions, etc.

\section{Conclusions}

The principle of least action for a networked complex system (eq. 1) drives selforganization in complex systems and the average action is the measure of degree to which they approach this least action state. Actions that are less than their alternatives are self-selected. Progressive development, as self-organization, is a process of minimization of action. In open systems there is a constant change of the number of elements, constraints and energy of the system and the least action state is different in each moment. The process of self-organization of energy, particles, atoms, molecules, organisms, to the today's society is a process of achieving a lower action state, with the least action as a final state. The laws of achieving this least action state are the laws of self-organization. The least possible action state is the limit for organization when time is infinite and all elements in the universe are included.

The state of nodes, edges, constraints and elements that determines the action for one motion in a system is its organization. With its measure $\alpha$ (eq. 2) we can compare any two systems of any size and the same system at two stages of its development. It distinguishes between systems with two different levels of organization and rates of self-organization and is normalized for their size. The measure can be applied to all systems and researchers in all areas studying complex systems can benefit from it. With a quantitative measure we can conduct exact scientific research on selforganization of complex systems and networks, progressive development, evolution and co-evolution, complexity, etc. 
Acknowledgments. The author thanks Assumption College for support and encouragement of this research, and Prof. Slavkovsky, Prof. Schandel, Prof. Sholes, Prof. Theroux and Prof. Cromarty for discussion of the manuscript. The author thanks Francis M. Lazarus, Eric Chaisson, John Smart, Clement Vidal, Arto Annila, Tessaleno Devezas, Yaneer Bar-Yam and Atanu Chatterjee for invaluable discussions.

\section{References}

1. Georgiev, G., Georgiev, I.: The least action and the metric of an organized system. Open Syst. Inf. Dyn. 9(4), 371 (2002)

2. de Maupertuis, P.: Essai de cosmologie (1750)

3. Goldstein, H.: Classical Mechanics. Addison Wesley (1980)

4. Feynman, R.: The Principle of Least Action in Quantum Mechanics. Ph.D. thesis (1942)

5. Gauss, J.: Über ein neues allgemeines Grundgesetz der Mechanik (1831)

6. Taylor, J.: Hidden unity in nature's laws. Cambridge University Press (2001)

7. Kaku, M.: Quantum Field Theory. Oxford University Press (1993)

8. Hoskins, D.A.: A least action approach to collective behavior. In: Parker, L.E. (ed.) Proc. SPIE, Microrob. and Micromech. Syst., vol. 2593, pp. 108-120 (1995)

9. Piller, O., Bremond, B., Poulton, M.: Least Action Principles Appropriate to Pressure Driven Models of Pipe Networks. In: ASCE Conf. Proc. 113 (2003)

10. Willard, L., Miranker, A.: Neural network wave formalism. Adv. in Appl. Math. 37(1), 19-30 (2006)

11. Wang, J., Zhang, K., Wang, E.: Kinetic paths, time scale, and underlying landscapes: A path integral framework to study global natures of nonequilibrium systems and networks. J. Chem. Phys. 133, 125103 (2010)

12. Annila, A., Salthe, S.: Physical foundations of evolutionary theory. J. Non-Equilib. Thermodyn., 301-321 (2010)

13. Devezas, T.C.: Evolutionary theory of technological change: State-of-the-art and new approaches. Tech. Forec. \& Soc. Change 72, 1137-1152 (2005)

14. Chaisson, E.J.: The cosmic Evolution. Harvard (2001)

15. Bar-Yam, Y.: Dynamics of Complex Systems. Addison Wesley (1997)

16. Smart, J.M.: Answering the Fermi Paradox. J. of Evol. and Tech. (June 2002)

17. Vidal, C.: Computational and Biological Analogies for Understanding Fine-Tuned Parameters in Physics. Found. of Sci. 15(4), 375-393 (2010)

18. Gershenson, C., Heylighen, F.: When Can We Call a System Self-Organizing? In: Banzhaf, W., Ziegler, J., Christaller, T., Dittrich, P., Kim, J.T. (eds.) ECAL 2003. LNCS (LNAI), vol. 2801, pp. 606-614. Springer, Heidelberg (2003) 